\title{
Laparoscopic-assisted instillation of epinephrine and levobupivacaine enables cornual excision and anatomical reconstruction in unruptured cornual pregnancy
}

\author{
Juan Gilabert-Estelles ${ }^{1,2 *}$, Juan Gilabert-Aguilar ${ }^{3,4}$ \\ ${ }^{1}$ Maternoinfantil Department, Hospital General Universitario, Valencia, Spain \\ ${ }^{2}$ Department of Pediatria, Obstetricia y Ginecologia, Universidad de Valencia, Valencia, Spain \\ ${ }^{3}$ Service of Gynecology, Hospital Arnau de Vilanova, Valencia, Spain \\ ${ }^{4}$ Universidad Católica San Vicente Mártir, Valencia, Spain \\ Email: “juangilaeste@yahoo.es
}

Received 26 December 2013; revised 13 January 2014; accepted 21 January 2014

Copyright (C) 2014 Juan Gilabert-Estelles, Juan Gilabert-Aguilar. This is an open access article distributed under the Creative Commons Attribution License, which permits unrestricted use, distribution, and reproduction in any medium, provided the original work is properly cited. In accordance of the Creative Commons Attribution License all Copyrights (c) 2014 are reserved for SCIRP and the owner of the intellectual property Juan Gilabert-Estelles, Juan Gilabert-Aguilar. All Copyright (C) 2014 are guarded by law and by SCIRP as a guardian.

\section{ABSTRACT}

The objective of this report is to describe the possible use of intramiometrial vasoconstrictive agents for laparoscopic management of interstitial pregnancy and the consequences in anatomical results and reproductive outcomes. Cornual resection can be performed by laparoscopy, but the high vascularization of this area may result in profuse bleeding and laparoscopic suturing under these conditions might be impossible for the majority of the surgeons. We present a case that describes the possible use of intramiometrial instillation of a solution of diluted epinephrine and levobupivacaine under laparoscopic guidance that permitted a bloodless cornual excision with complete reconstruction. Vasoactive agents might have potentially serious cardiovascular side effects and the correct election of the active principle and the dosage is essential to reduce the risk of the surgery and obtain good anatomical results and reproductive outcomes. In conclusion, unruptured interstitial pregnancies can be managed successfully with intramyometrial instillation of epinephrine and bupivacaine. This simple technique is particularly attractive as it facilitates anatomical reconstruction of the cornual area, gives enough time to perform a complete suture of the defect and reduces the risk of laparotomic conversion.

\section{KEYWORDS}

Cornual Pregnancy; Levobupivacaine; Epinephrine; Laparoscopy; Vasoconstrictive Agents

"Corresponding author.

\section{INTRODUCTION}

Cornual pregnancy is a rare entity that affects up to $4 \%$ of women suffering a tubal pregnancy [1]. It is defined as the presence of a gestational sac in the interstitial portion of the fallopian tube, which is embodied within the muscular wall of the uterine horn. The special location of this type of ectopic pregnancy, in a high vascularised anatomic area, may lead to a life-threatening clinical presentation. Maternal mortality has been reported to be as high as $2 \%-2.5 \%$ [1], mainly due to hemodynamic complications after the rupture of the uterus in delayed diagnosed cases. Classical treatment of interstitial pregnancy included cornual resection by laparotomy or even hysterectomy if it is associated with severe uterine damage. Minimal invasive approach by laparoscopy has been used for more than two decades for the management of cornual pregnancies [2]. Nevertheless, the risk of bleeding and the difficulties encountered during suturing the anatomical defect are still the main concerns for laparoscopic surgeons. Multiple techniques have been reported in order to achieve a better hemostasis but specific instrumentation is usually required and thermal damage might reduce the quality of the uterine scar. We describe the simple use of an intramiometrial combination of vasoconstrictive agents with a two-layer closure with laparoscopic suture for the management of interstitial pregnancy comparing the different methods to control haemorrhage reported in the literature.

\section{MATERIAL AND METHODS}

We report our experience in a patient with a cornual 
pregnancy who was successfully managed with a simple inexpensive technique that included infiltration of a solution with diluted epinephrine and levobupivacaine and right cornual wedge resection with salpingectomy. Anatomical reconstruction of the cornual area was achieved by a two-layer intracorporeal suturing under laparoscopic guidance.

We also conducted a MEDLINE search from January 1970 to December 2010 using combinations of the medical subject headings "cornual”, "interstitial”, “intramural”, and "ectopic pregnancy", retrieving a total of 126 articles and reviews. Only pertinent articles that related with the specific laparoscopic management and different techniques for reducing bleeding at the cornual area were considered. Additional reports, including those referring specifically to the use of vasoconstrictive agents in gynecology were also considered.

\section{RESULTS}

A 38-year-old woman with no previous surgical procedures attended our unit, with abdominal pain and spotting 8 weeks after her last menstrual period. At presentation the patient's general condition was good and hemodynamically stable. The patient had no comorbid conditions and she referred no previous surgery. Abdominal examination revealed tenderness and intestinal peristalsis was identified on auscultation. Gynecological examination revealed painful cervix mobilization and augmented right adnexa. The ultrasound exam showed a right adnexal mass of $3 \times 4 \mathrm{~cm}$ with an embryo of $7 \mathrm{~mm}$ that presented positive heart beating that appeared to be located at the proximal portion of the right tube. Routine blood tests were taken showing no signs of anemia or infection. Serum B-hCG was 3238 mU/mL. A laparoscopic removal of the ectopic pregnancy was indicated. Laparoscopic access was performed using a visual port insertion with Ternamian endotip trocar (Karl Storz, Tuttlingen, Germany). At examination there was an unruptured mass of $4 \times 3 \mathrm{~cm}$ that seemed to origin from the right horn of the uterus. The right salpinx and the right ovary were normal. Left ovary and tube were also normal. The uterus was enlarged and plump. It was normal except for some dense adhesions in the posterior uterine surface. Intramyometrial instillation of a $50 \mathrm{~mL}$ solution with diluted epinephrine and levobupivacaine was used in order to permit a bloodless cornual excision. The solution was prepared by an assistant in a 50-mL syringe connected to a laparoscopic cannula. The syringe contained $50 \mathrm{~mL}$ of Levobupivacaine chloride $0.25 \%$ (Chirocane Sol. iny. 0.25\%, Abbot Lab. SA, España) and $0.25 \mathrm{~mL}$ of epinephrine ([1/4] vial of $1 \mathrm{mg} / \mathrm{mL})$. The infiltration was performed deeply inside the cornual myometrium, medially to the limit of the ectopic pregnancy, to avoid the direct infiltration inside the chorial tissue.
Repeated aspirations were performed to prevent intravascular injection before infiltration and the systolic and diastolic blood pressure and heart rate were accurately controlled by the anesthesiologist. An elliptic incision was made above the base of the cornual pregnancy, leaving enough serosa and myometrium for closure the defect and a wedge resection at the cornual area was performed. Very scant bleeding was noted during removal of the products of gestation, which facilitated the anatomical reconstruction. The defect was first sutured with a two-layer introflecting stitch in order to prevent slippage. Coagulation was avoided in order to preserve the quality of the uterine scar and to avoid damaging the endometrium. A nitrocellulose barrier (Gynecare Interceed, Johnson \& Johnson, NY, USA) was left in the surgical area in order to prevent adhesions. The products of gestation were removed laparoscopically using an endoscopic bag (Endocatch Gold, 10 mm; Covidien, Norwalk, CT, USA).

During the next two days of hospitalization the patient was in a good general condition without any pain in the abdomen. The routine palpatory and auscultatory examinations revealed no abnormalities. The postoperative period was uneventful. Hemoglobin drop was $0.8 \mathrm{~g} / \mathrm{dL}$ and control serum beta-hCG 24 hours after surgery was $943 \mathrm{mU} / \mathrm{ml}$, and was repeated weekly after neutralization 14 days later. The patient was discharged 48 hours after the laparoscopic procedure without further complications. Postoperative gynaecological examination performed one month later was normal and at the present the patient is not seeking pregnancy.

\section{DISCUSSION}

Since Reich reported the first interstitial pregnancy managed by laparoscopy more than two decades ago [2], many laparoscopic techniques for the treatment of this entity have been described. The laparoscopic removal of the products of gestation has become the standard treatment of tubal ectopic pregnancy. The chances to perform a conservative management of the interstitial pregnancy depend on the delay of diagnosis. The procedure usually starts with a salpingectomy and removing the cornual pregnancy using bipolar forceps and scissors. Usually hemostasis is achieved using electrocoagulation [3] but other techniques have been tested such as fibrin glue [4] , harmonic scalpel [5], cornuostomy after temporary tourniquet [6], selective ipsilateral uterine artery ligation [7] or combined laparoscopic and hysteroscopic approach [8]. Ostrzenski et al. also described the effective use of a ligature applied through the broad ligament [9]. Endoscopic stapler devices have also been tested to control cornual bleeding in complicated interstitial pregnancy [10] but they arise some concerns, such as the possible limited effectiveness in bulky tissues, such as myo- 
metrium.

Several vasoconstrictive agents have been described in order to control haemorrhage during gynaecological procedures when opening the myometrium. Vassopresin (8l-arginine vasopressin) is the most frequently vasoconstrictive agent used to reduce the blood loss in the English literature [11-15]. Nevertheless, vassopresin is not approved in several countries, including our country, due to the potentially serious side effects reported in the literature that may cause lethal cardiopulmonary complications [16-19]. Other vasoconstrictive agents have been used in gynaecological procedures. Zullo et al. [20] performed a randomized study to evaluate the effectiveness of the injection of bupivacaine plus epinephrine before laparoscopic myomectomy. They encountered a reduction in the blood loss, the global operative and enucleation times, the subjective surgical difficulty and the postoperative pain. A systematic review of randomized trials in which hemorrhage during myomectomy was evaluated showed the effectiveness of vaginal misoprostol, intramyometrial vasopressin and analogues, intramyometrial bupivacaine plus epinephrine and pericervical tourniquet. Nevertheless, no evidence of effect with myoma enucleation by morcellation and oxytocin was demonstrated [21]. Epinephrine is a potent vasoconstrictive agent that has a high risk of cardiovascular effects if an intravascular instillation is performed. As the cornual area is highly vascularizated it is important to minimize the dose of epinephrine without hampering the vasospasm in order to obtain a bloodless cornuostomy. The vasoconstrictive effect of epinephrine on tissue lasts longer than that of vasopressin (5 - 6 hours versus 17 - 35 minutes) [22], which might result in inaccurate hemostasis if a complete suture of the defect is not performed. In addition, high doses of epinephrine can induce long vasoconstriction inducing tissue ischemia and impairing the healing process [23].

Bupivacaine and levobupivacaine are local anesthetics that cause vasodilatation at clinical doses, but lower doses appear to cause vasoconstriction. Levobupivacaine has shown to have vasoconstrictive activity in concentrations of $\leq 0.25 \%$, with duration of its action between 4 and $24 \mathrm{~h}$ when used for local infiltration [24]. In addition, levobupivacaine has similar potency to bupivacaine with a lower risk of cardiovascular and nervous toxicity, which makes it a good election for its anesthetic and vasoconstrictive properties [25]. Clinical studies have tested the efficacy of levobupivacaine in a wide spectrum of operations, such as surgical wound infiltration or intraperitoneal instillation after laparoscopic procedures [26, 27]. We decided to use a combination of levobupivacaine and low dose of epinephrine in order to minimize the cardiovascular effects of the latest without interfering in the vasoconstrictive effects on hemostasis.

Medical treatment with methotrexate in women wish- ing to preserve their reproductive capabilities has been described since more than two decades [28,29]. Nevertheless, medical treatment failure has been reported in $12 \%$ of cases of interstitial ectopic pregnancies [30]. Moreover, there are no reliable clinical measures that may predict the successful response to methotrexate. Lau and Tulandi [31] found lower serum B-hCG levels in patients who failed to respond adequately to methotrexate and subsequently required surgery. This may suggest that serum B-hCG levels in interstitial pregnancy might not be predictive of treatment success. In addition, limited information is provided in the literature concerning the size of the interstitial pregnancy and therefore no prediction on the therapy response can be made on this measurement. Ross et al. [32] reported a novel treatment regimen in order to decrease the possible risk of hemorrhage that combined the evacuation of the gestation under sonographic guidance, leaving the placenta in situ, and a postoperative methotrexate. In the present report, embryo cardiac activity was present, which may be in relation to a high chorial activity. The intraoperative finding of a non-ruptured cornual pregnancy led us to adopt a definitive therapy that included removal of all the chorial tissue with anatomical reconstruction of the defect. In addition, first-line complete resolution of the case might be of special interest in women over 35-year-old that may wish to conceive in the future or might be candidates for assisted reproductive techniques.

\section{CONCLUSION}

In conclusion, our experience suggests that unruptured interstitial pregnancies can be managed successfully with intramyometrial instillation of epinephrine and levobupivacaine under laparoscopic guidance. This technique is particularly attractive as it facilitates anatomical reconstruction of the cornual area, gives enough time to perform a complete suture of the defect and reduces the risk of laparotomic conversion.

\section{CONFLICT OF INTEREST}

None declared.

\section{REFERENCES}

[1] Tulandi, T. and Al-Jaroudi, D. (2004) Interstitial pregnancy: Results generated from the Society of Reproductive Surgeons Registry. ACOG, 103, 47-50.

[2] Reich, H., McGlynn, F., Budin, R., Tsoutsoplides, G. and DeCaprio, J. (1990) Laparoscopic treatment of ruptured interstitial pregnancy. Journal of Gynecologic Surgery, 6, 135-138. http://dx.doi.org/10.1089/gyn.1990.6.135

[3] Tinelli, A., Malvasi, A., Pellegrino, M., Pontrelli, G., Martulli, B. and Tsin, D.A. (2010) Laparoscopical management of cornual pregnancies: A report of three cases. 
European Journal of Obstetrics \& Gynecology and Reproductive Biology, 151, 199-202.

http://dx.doi.org/10.1016/j.ejogrb.2010.03.032

[4] Morita, Y., Tsutsumi, O., Momoeda, M. and Taketani, Y. (1997) Cornual pregnancy successfully treated laparoscopically with fibrin glue hemostasis. Obstetrics \& Gynecology, 90, 685-687.

http://dx.doi.org/10.1016/S0029-7844(97)00398-0

[5] Dalkalitsis, N., Stefanidis, K., Paschopoulos, M., Navrozoglou, I., Mouzakioti, E. and Lolis, D. (1998) Laparoscopic treatment of interstitial pregnancy using the harmonic scalpel. Clinical \& Experimental Obstetrics \& Gynecology, 25, 49-50.

[6] Choi, Y.S., Eun, D.S., Choi, J., Shin, K.S., Choi, J.H. and Park, H.D. (2009) Laparoscopic cornuotomy using a temporary tourniquet suture and diluted vasopressin injection in interstitial pregnancy. Fertility and Sterility, 91, 19331937. http://dx.doi.org/10.1016/j.fertnstert.2008.02.013

[7] Raheem, M. and Yousri Afifi, Y. (2008) Laparoscopic selective ipsilateral uterine artery ligation for the management of a cornual ectopic pregnancy. Journal of Minimally Invasive Gynecology, 15, 260-261. http://dx.doi.org/10.1016/j.jmig.2008.02.008

[8] Katz, D.L., Barrett, J.P., Sanfilippo, J.S. and Badway, D.M. (2003) Combined hysteroscopy and laparoscopy in the treatment of interstitial pregnancy. American Journal of Obstetrics \& Gynecology, 188, 1113-1114. http://dx.doi.org/10.1067/mob.2003.258

[9] Ostrzenski, A. (1997) A new laparoscopic technique for interstitial pregnancy resection. A case report. The Journal of Reproductive Medicine, 42, 363-366.

[10] Sergent, F., Le Cornec, J.B., Meilhaud, M.F. and Marpeau, L. (2003) Laparoscopic cornual excision with an automatic stapler for ruptured interstitial pregnancies. Journal de Gynecologie, Obstetrique et Biologie de la Reproduction's, 32, 426-430.

[11] Moon, H.S., Choi, Y.J., Park, Y.H. and Kim, S.G. (2000) New simple endoscopic operations for interstitial pregnancies. American Journal of Obstetrics \& Gynecology, 182, 114-121. http://dx.doi.org/10.1016/S0002-9378(00)70499-6

[12] Schulz, K.F., Grimes, D.A. and Christensen, D.D. (1985) Vasopressin reduces blood loss from second-trimester dilatation and evacuation abortion. Lancet, 2, 353-356. http://dx.doi.org/10.1016/S0140-6736(85)92496-1

[13] Fletcher, H., Frederick, J., Hardie, M. and Simeon, D. (1996) A randomized comparison of vasopressin and tourniquet as hemostatic agents during myomectomy. Obstetrics \& Gynecology, 87, 1014-1018. http://dx.doi.org/10.1016/0029-7844(96)00071-3

[14] Phillips, D.R., Nathanson, H.G., Milim, S.J., Haselkorn, J.S., Khapra, A. and Ross, P.L. (1996) The effect of dilute vasopressin solution on blood loss during operative hysteroscopy: A randomized controlled trial. Obstetrics \& Gynecology, 88, 761-766. http://dx.doi.org/10.1016/0029-7844(96)00282-7

[15] Okin, C.R., Guido, R.S., Meyn, L.A. and Ramanathan, S. (2001) Vasopressin during abdominal hysterectomy: A randomized controlled trial. Obstetrics \& Gynecology, 97, 867-872.

http://dx.doi.org/10.1016/S0029-7844(01)01353-9

[16] Nezhat, F., Admon, D., Nezhat, C.H., Dicorpo, J.E. and Nezhat, C. (1994) Life-threatening hypotension after vasopressin injection during operative laparoscopy, followed by uneventful repeat laparoscopy. Journal of the American Association of Gynecologic Laparoscopists, 2, 83-86. http://dx.doi.org/10.1016/S1074-3804(05)80837-0

[17] Tulandi, T., Beique, F. and Kimia, M. (1996) Pulmonary edema: A complication of local injection of vasopressin at laparoscopy. Fertility and Sterility, 66, 478-480.

[18] Lurie, S. and Mamet, Y. (2000) Transient myocardial ischemia may occur following subendometrial vasopressin infiltration. European Journal of Obstetrics \& Gynecology and Reproductive Biology, 91, 87-89. http://dx.doi.org/10.1016/S0301-2115(99)00233-X

[19] Rutsuko, H., Sachiko, N., Yasuki, K. and Osamu, T. (2009) Bradycardia and Cardiac Arrest Caused by Intramyometrial Injection of Vasopressin during a Laparoscopically Assisted Myomectomy. Obstetrics \& Gynecology, 113, 484-486.

[20] Zullo, F., Palomba, S., Corea, D., Pellicano, M., Russo, T., Falbo, A., Barletta, E., Saraco, P., Doldo, P. and Zupi, E. (2004) Bupivacaine plus epinephrine for laparoscopic myomectomy: A randomized placebo-controlled trial. Obstetrics \& Gynecology, 104, 243-249.

http://dx.doi.org/10.1097/01.AOG.0000132801.41880.e8

[21] Kongnyuya, E.J., van den Broeka, N. and Wiysongeb, C.S. (2008) A systematic review of randomized controlled trials to reduce hemorrhage during myomectomy for uterine fibroids. Obstetrics \& Gynecology, 100, 4-9.

[22] McClellan, K.J. and Spencer, C.M. (1998) Levobupivacaine. Drugs, 56, 355-362. http://dx.doi.org/10.2165/00003495-199856030-00005

[23] England, G.T., Randall, H.W. and Graves, W.L. (1983) Impairment of tissue defenses by vasoconstrictors in vaginal hysterectomies. Obstetrics \& Gynecology, 61, 271274.

[24] Newton, D.J., McLeod, G.A., Khan, F. and Belch, J.J. (2005) Vasoactive characteristics of bupivacaine and levobupivacaine with and without adjuvant epinephrine in peripheral human skin. British Journal of Anaesthesia, 94, 662-667. http://dx.doi.org/10.1093/bja/aei095

[25] Foster, R.H. and Markham, A. (2000) Levobupivacaine: A review of its pharmacology and use as a local anaesthetic. Drug, 59, 551-579. http://dx.doi.org/10.2165/00003495-200059030-00013

[26] Louizos, A.A., Hadzilia, S.J., Leandros, E., Kouroukli, I.K., Georgiou, L.G. and Bramis, J.P. (2005) Postoperative pain relief after laparoscopic cholecystectomy: A placebo-controlled double-blind randomized trial of preincisional infiltration and intraperitoneal instillation of levobupivacaine 0.25\%. Surgical Endoscopy, 19, 1503-1506. http://dx.doi.org/10.1007/s00464-005-3002-4

[27] Alessandri, F., Lijoi, D., Mistrangelo, E., Nicoletti, A. and Ragni, N. (2006) Effect of presurgical local infiltration of levobupivacaine in the surgical field on postsur- 
gical wound pain in laparoscopic gynecological surgery. Acta Obstetricia et Gynecologica Scandinavica, 85, 844849. http://dx.doi.org/10.1080/00016340500494846

[28] Benifla, J.L., Fernandez, H., Sebban, E., Darai, E., Frydman, R. and Madelenat, P. (1996) Alternative to surgery of treatment of unruptured interstitial pregnancy: 15 cases of medical treatment. European Journal of Obstetrics \& Gynecology and Reproductive Biology, 70, 151-156. http://dx.doi.org/10.1016/S0301-2115(95)02589-8

[29] Lalchandani, S., Geary, M., O’Herlihy, C. and Sheil, O. (2003) Conservative management of placenta accreta and unruptured interstitial cornual pregnancy using methotrexate. European Journal of Obstetrics \& Gynecology and Reproductive Biology, 107, 96-97. http://dx.doi.org/10.1016/S0301-2115(02)00241-5
[30] Fisch, J.D., Ortiz, B.H., Tazuke, S.L., Chitkara, U. and Giudice, L.C. (1998) Medical management of interstitial ectopic pregnancy: A case report and literature review. Human Reproduction, 7, 1981-1986. http://dx.doi.org/10.1093/humrep/13.7.1981

[31] Lau, S. and Tulandi, T. (1999) Conservative medical and surgical management of interstitial ectopic pregnancy. Fertility and Sterility, 72, 207-215. http://dx.doi.org/10.1016/S0015-0282(99)00242-3

[32] Ross, R., Lindheim, S.R., Olive, D.L. and Pritts, E.A. (2006) Cornual gestation: A systematic literature review and two case reports of a novel treatment regimen. JMIG, 13, 74-78. 